\title{
The RNA-Binding Protein RBM3 Is Required for Cell Proliferation and Protects Against Serum Deprivation-Induced Cell Death
}

\author{
SVEN WELLMANN, MATTHIAS TRUSS, ELISABETH BRUDER, LUIGI TORNILLO, ANDREA ZELMER, KARL SEEGER, \\ AND CHRISTOPH BÜHRER
}

Clinic of Neonatology [S.W.], University Hospital Zürich, CH-8091 Zürich, Switzerland; Departments of Pediatrics [M.T.], Pediatric Oncology and Hematology [K.S.], and Neonatology [C.B.], Charité University Medical Center, D-13344 Berlin, Germany; Institute of Pathology [E.B., L.T.], Basel University Hospital, CH-4003 Basel, Switzerland; Basel University Children's Hospital [A.Z., C.B.], CH-4005 Basel, Switzerland

\begin{abstract}
Hypoxia and other adverse conditions are commonly encountered by rapidly growing cells. The RNA-binding protein RBM3 (RNA-binding motif protein 3), which is transcriptionally induced by low temperature and hypoxia, has recently been implicated in survival of colon cancer cells by mechanisms involving cyclooxygenase-2 (COX-2) signaling. Immunohistochemically, we found strong RBM3 expression in a variety of malignant and proliferating tissues but low expression in resting and terminally differentiated cells. RBM3 expression in fibroblasts and human embryonal kidney (HEK293) cells subjected to serum deprivation or contact inhibition closely paralleled proliferation rates, assessed by real-time RT-PCR and immunoblotting. siRNA-mediated RBM3 knockdown reduced cell viability and finally led to cell death, which did not involve caspase-3-mediated apoptosis, cell cycle arrest, or COX-2 regulation. In contrast, RBM3 over-expression rescued cells from death under serum starvation. This was associated with increased translation rates, as measured by ${ }^{14} \mathrm{C}$ serine and ${ }^{3} \mathrm{H}$ phenylalanine incorporation. Together, RBM3 is a critical factor providing cellular survival advantages in an adverse microenvironment presumably by restoring translation efficacy. (Pediatr Res 67: 35-41, 2010)
\end{abstract}

$\mathrm{S}^{\mathrm{n}}$ plicing, stability and transport of mRNA, and ultimately mRNA translation into proteins are tightly regulated by RNA-binding proteins. The RNA-binding motif protein 3 (RBM3) is an evolutionary highly conserved RNA binding protein of 157 amino acids with a predicted mass of $17 \mathrm{kD}$ (1). We have previously demonstrated that both RBM3 mRNA and protein levels increase in response to hypoxia in a hypoxia inducible factor (HIF)-1-independent fashion (2). RBM3 is also one of the first proteins synthesized in response to cold shock $(3,4)$ and consistently elevated during winter in hibernating animals, such as the ground squirrel (5).

An involvement of RBM3 in the regulation of translation has been deduced from increased rates of protein synthesis associated with its over-expression in transfected cells or RBM3 up-regulation after hypothermia (6). Over-expression of RBM3 has also been shown to suppress cell death in cells

Received May 13, 2009; accepted September 6, 2009

Correspondence: Sven Wellmann, M.D., Klinik für Neonatologie, UniversitätsSpital Zürich, Frauenklinikstr. 10, CH-8091 Zürich, Switzerland; e-mail: sven.wellmann@usz.ch

Supported by grants from the Wilhelm-Sander-Foundation (2004.162.1), Munich, Germany, and the Bangerter-Rhyner-Foundation, Bern, Switzerland. harboring polyglutamine-huntingtin (7) and to stabilize cyclooxygenase-2 (COX-2) mRNA transcripts (8). Moreover, RBM3 was found to prevent cells from caspase-mediated apoptosis and mitotic catastrophe (9). On the other hand, down-regulation of RBM3 in human colon cancer cells has been reported to cause loss of COX-2 translation and decrease cell growth in culture, which was partially overcome with the COX-2 product, prostaglandin E2 (9).

Here, we set out to further characterize the link between RBM3, cell proliferation, and apoptosis. We found that RBM3 expression paralleled proliferation rates in vitro and in vivo. Over-expression of RBM3 rescued human embryonal kidney (HEK293) cells from cell death triggered by serum starvation, and siRNA-mediated RBM3 knockdown-induced cell death occurred without caspase-3 activation. Both artificial up- and down-regulation of RBM3 in HEK293 cells had no effect on COX-2 expression, indicating that other mechanisms are involved that improve cell survival especially under adverse conditions.

\section{MATERIALS AND METHODS}

Cell lines, lymphocyte preparation, culture conditions, and treatment. HEK293, human colon adenocarcinoma (HT29), and human chronic myeloid leukemia (K562) cells were purchased from the German Collection of Microorganisms and Cell cultures (DSMZ, Braunschweig, Germany, http://www.dsmz.de/). The human fibroblast cell line MRC-5 was a gift from Dr. G. Orend (Institute of Biochemistry and Genetics, University of Basel, Switzerland). HEK293, HT29, and MRC-5 cells were grown in DMEM and K562 cells in RPMI 1640, both the mediums supplemented with $10 \%$ heat inactivated FCS and 100 units penicillin/streptomycin per $\mathrm{mL}$ (all from Invitrogen, Basel, Switzerland), at $37^{\circ} \mathrm{C}$ and $5 \% \mathrm{CO}_{2}$ in a fully humidified incubator. Primary human lymphocytes were isolated from peripheral blood as described previously (10). Lymphocytes were treated and stimulated as indicated with the proteasome inhibitor MG132, IL-2, or phytohemagglutinin (PHA, all Sigma Chemical Co--Aldrich, Buchs, Switzerland). For inhibition of COX-2 signaling, two inhibitors indomethacin and the selective COX-2 inhibitor indomethacin heptyl ester (both Sigma Chemical Co.-Aldrich) were used. TNF- $\alpha$ was used as indicated (Sigma Chemical Co.-Aldrich).

Real-time RT-PCR analyses. Real-time RT-PCR was performed as previously described $(2,11)$. All primers were purchased from Microsynth (Microsynth, Balgach, Switzerland), and sequences are given in Table 1.

\footnotetext{
Abbreviations: COX-2, cyclooxygenase-2; HEK293, human embryonal kidney; HT29, human colon adenocarcinoma cells; K562, human chronic myeloid leukemia cells; MTA, multitissue Array; RBM3, RNA-binding motif protein 3
} 
Table 1. Primers used for cDNA amplification

\begin{tabular}{lll}
\hline & \multicolumn{1}{c}{ Forward primer } & \multicolumn{1}{c}{ Reverse primer } \\
\hline RBM3 & $5^{\prime}$-CTTCAGCAGTTTCGGACCTA & $5^{\prime}$-ACCATCCAGAGACTCTCCGT \\
CyclinD1 & $5^{\prime}$-CTGGCCATGAACTACCTGGA & $5^{\prime}$-GTCACACTTGATCACTCTGG \\
$\beta$-2-microglobulin & $5^{\prime}$-GATGAGTATGCCTGCCGTGTG & $5^{\prime}$-TCCAATCCAAATGCGGCATCT \\
$\beta$-actin & $5^{\prime}$-AGCCTCGCCTTTGCCGA & $5^{\prime}$-CTGGTGCCTGGGGCG \\
COX-2 & $5^{\prime}$-CCTTCCTCCTGTGCCTGATG & $5^{\prime}$-ACAATCTCATTTGAATCAGGAAGCT \\
COX-1 & $5^{\prime}$-TGTTCGGTTCCAGTTCCAATA & $5^{\prime}$-ACCTTGAAGGAGTCAGGCATGAG \\
IL-8 & $5^{\prime}$-CTTGGCAGCCTTCCTGATTT & $5^{\prime}$-TCCAGACAGAGCTCTCTTCCATC \\
VEGF & 5'-CCCTGATGAGATCGAGTACATCTT & $5^{\prime}$-CTTGTCTTGCTCTATCTTTCTTTGGTCT \\
\hline
\end{tabular}

Protein extraction and immunoblot analyses. Differential nuclear und cytosolic extracts from HEK293 and K562 cells were prepared by use of the TRansFactor Extraction Kit according to the manufactures recommendations (BD Biosciences, Allschwil, Switzerland). Total cell protein extraction, quantification, and immunoblotting were performed as described previously $(2,12)$ using rabbit polyclonal antibodies against human RBM3 [kindly provided by Dr. Jun Fujita Department of Clinical Molecular Biology, Kyoto University, Kyoto 606-8507, Japan (2)], Caspase-3, Cleaved Caspase-3 (Asp175), or mouse MAb against CyclinD1 (DCS6, all from Cell Signaling, Danvers, MA,) or against COX-2 (Cayman Chemical, Ann Arbor, MI). As an internal control, blots were stripped and reprobed with mouse anti- $\beta$-actin antibody (Sigma Chemical Co.-Aldrich). Bound antibodies were detected using secondary antibodies conjugated with horseradish peroxidase (Cell Signaling) and enhanced chemiluminescence systems (ECL, Amersham).

RBM3 over-expression. The sequence of the RBM3 gene GenBank NM_006743 was optimized for increased mammalian expression, resynthesized chemically by GENEART (GENEART company, Regensburg, Germany), and cloned into the expression vector pCEP4, containing a hygromycin resistance gene (Invitrogen). HEK293 cells were transfected either with the RBM3 containing vector or the empty vector, serving as control, by use of Nanofectamine reagent (PAA Laboratories, Cölbe, Germany) according to the manufacturer's instructions. Stable overexpressing cell pools were established by cell growth in selective medium (10\% FCS-DMEM containing hygromycin-B $150 \mu \mathrm{g} / \mathrm{mL}$, a concentration that caused $100 \%$ cell death of nontransfected HEK293 cells within 1-2 wk). Screening for successful transfection was done by western blotting analysis for RBM3 protein levels, as described below.

siRNA. For transient down-regulation of target mRNAs, various siRNA sequences were used targeting the coding region of RBM3 (GenBank NM_006743): RBM3-A 5'-GTGGATCATGCAGGCAAGT-3', RBM3-B 5' GGTGGTTATGACCGCTACT-3', RBM3-C 5'-CAGGAGGAAATTACAGAGACA-3', RBM3-D 5'-CCAAATGGCTGTATTTATAAA-3', RBM3-E 5'-TAGGTTTATTGGAGCTAGCAA-3'. siRNAs targeting mRNAs of various control mRNAs served as controls enhanced green fluorescent protein (EGFP) 5'-GCAGCACGACTTCTTCAAG-3', Luc (directed against firefly luciferase; pGL2 vectors; Promega, Madison, WI) 5'-CGTACGCGGAATACTTCGA-3', and E2F transcription factor 4 (E2F4) 5'-GCGGCGGATTTACGACATT-3' (13). For siRNA experiments, HEK293-cells were grown to $50 \%$ confluent in poly-D-lysine coated 12-well microplates (Becton Dickinson, Basel, Switzerland) and transfected with siRNAs using Nanofectin siRNA reagent (PAA Laboratories, Pasching, Austria) according to the manufacturer's instructions. For stable down-regulation of target mRNAs, two RBM3 siRNA sequences (RBM3-A and -B) as well as E2F4 and EGFP for control were cloned in the previously characterized episomal siRNA expression plasmid vector pRep-H1 (13). HEK293 cells were transfected by use of the Nanofectamine reagent (PAA Laboratories) and K562 cells by electroporation as described (13). Twenty-four hours after transfection, cells were placed in selective medium containing puromycin $4 \mu \mathrm{g} / \mathrm{mL}$, a concentration that caused $100 \%$ cell death of nontransfected HEK293 and K562 cells within 1-2 wk.

Cell viability and growth measurements. The viability of cells was examined by the use of the MTS [3-(4,5-dimethylthiazol-2-yl)-5-(3carboxymethoxyphenyl)-2-(4-sulfophenyl)-2H-tetrazolium] tetrazolium assay according to the manufacturers recommendations (Cell Titer96 Aqueous, Promega, Dübendorf, Switzerland). A colorimetric immunoassay, based on Bromodeoxyuridine (5-bromo-2-deoxyuridine, BrdU) incorporation during DNA synthesis, was applied to quantify cell proliferation (Roche, Basel, Switzerland).

Protein synthesis. Protein synthesis was measured with few modifications as described previously (14). Briefly, $1 \times 10^{5}$ HEK293 cells per milliliter, either stable transfectants over-expressing RBM3 and controls or various siRNA transfectants, were plated into 12-well microplates and incubated in complete or starved medium as indicated. After $3 \mathrm{~d}$, cells were carefully washed and minimal medium supplemented with L-Serine-UL-14C (Sigma Chemical Co.-Aldrich) was added. After incubation at $37^{\circ} \mathrm{C}$ for $3 \mathrm{~h}$, cells were harvested by trypsinization, washed five times with ice-cold PBS, precipitated in 5\% ice-cold trichloroacetic acid, redissolved in $1 \mathrm{~N} \mathrm{NaOH}$, and neutralized by addition of $1 \mathrm{~N} \mathrm{HCl}$. Finally, aliquots were counted after addition of OptiPhase (Perkin Elmer, Waltham, MA) using a liquid scintillation analyzer (Perkin Elmer). Replicate wells within the same multiwell plate were harvested for both protein determination and cell counting as described earlier. In addition to L-Serine-UL-14C fixation, $1-[2,3,4,5,6-3 \mathrm{H}]$ Phenylalanine (Amersham) incorporation was also estimated as a metabolic control with similar results.

Flow cytometric cell cycle analysis. Cell cycle analysis including detection of apoptotic cells was performed as described previously (15). Data analysis was performed in WIN MDI 2.8 and the Cylchred program (see www.facslab.toxikologie.uni-mainz.de).

Construction of a paraffin tissue arrays. Two paraffin multitissue arrays (MTA) were constructed from various tissue punches entered into two receptor blocks, respectively, as previously described (16). Punch diameter was $0.6 \mathrm{~mm}$. The first MTA included a selection of 110 normal and tumor tissues as given in Table 2. The second MTA included 10 normal brain tissues and 255 CNS (CNS) tumors specimens as given in Table 3. Fourteen of 110 tissue punches in the first MTA and 20 of 265 punches in the CNS MTA were excluded from analysis because of missing material.

Immunohistochemistry. Paraffin tissue array sections were cut at $4 \mu \mathrm{m}$ and deparaffinized overnight in Xylene, rehydrated in descending ethanol series, and rinsed in PBS buffer. Antigen retrieval was achieved with pressure cooker for $5 \mathrm{~min}$ at $120^{\circ} \mathrm{C}$ in citrate buffer pH6 (ProTaqs 400300692), followed by washing in TBS/PBS (1:10) buffer. Endogenous peroxidase was quenched with incubation for $10 \mathrm{~min}$ in $3 \% \mathrm{H}_{2} \mathrm{O}_{2}$, followed by washing in TBS/PBS buffer. Incubation of primary rabbit polyclonal antibody against human RBM3 (2) was performed for $2 \mathrm{~h}$ at $30^{\circ} \mathrm{C}$ in a moist chamber at a dilution of 1:5000, washed twice in TBS/PBS buffer, then incubated for 30 min with Envision Polymer HRP (DAKO K4002) at $30^{\circ} \mathrm{C}$ in a moist chamber and finally washed twice in TBS/PBS buffer. Chromogen detection was achieved with rinse in Tris/distilled water (1:10) for 10 min and incubation for 5 min with DAB-Chromogen(Liquid DAB DAKO Code No.: K 3467). Slides were washed in demineralized water and counterstained for $20 \mathrm{~s}$ with Hematoxylin(Harris HTX 31000, Medite $\mathrm{GmbH}$ ), rinsed with water, differentiated in HCL-Ethanol and blued for $5 \mathrm{~min}$ in tap water, followed by dehydration in ascending ethanol series, mounting and coverslipping.

Immunohistochemically stained slides were evaluated by two experienced pathologists (L.T., E.B.). Grading was performed as follows: grading of staining intensity was done in a semiquantitative manner and noted as negative, weakly positive $(1+)$, moderately positive $(2+)$, and strongly positive $(3+)$. Subcellular localization of staining signal was noted as nuclear or cytoplasmic and grading of staining intensity was performed independently for both subcellular locations. In addition, for each staining signal, the percentage of cells expressing the antigen was noted individually (see Tables 2 and 3 ).

All experiments involving human samples had been approved by the local Institutional Review Board (Ethikkommission der Universität Basel).

\section{RESULTS}

RBM3 expression is strongly associated with cell proliferation in vitro. When confluent grown HEK293 cells were split and seeded in fresh medium, cell proliferation rates rose subsequently as measured by $\mathrm{BrdU}$ incorporation and $\mathrm{Cy}$ clinD1 expression (Fig. 1A). RBM3 mRNA and protein expression increased strongly alongside the increased prolifera- 
Table 2. RBM3 expression in normal and malignant tissues

\begin{tabular}{|c|c|c|c|c|c|}
\hline \multirow[b]{2}{*}{ Tissue } & \multirow[b]{2}{*}{ Sample } & \multicolumn{2}{|c|}{ Nuclear } & \multicolumn{2}{|c|}{ Cytoplasm } \\
\hline & & Intensity & $\%$ & Intensity & $\%$ \\
\hline \multirow[t]{4}{*}{ Adrenal } & 1 & + & 80 & + & 100 \\
\hline & 2 & - & 0 & - & 0 \\
\hline & 3 & + & 40 & - & 0 \\
\hline & 4 & ++ & 30 & - & 0 \\
\hline \multirow[t]{8}{*}{ Breast carcinoma } & 1 & +++ & 90 & + & 100 \\
\hline & 2 & +++ & 90 & + & 100 \\
\hline & 3 & ++ & 90 & - & 0 \\
\hline & 4 & + & 50 & - & 0 \\
\hline & 5 & +++ & 90 & + & 100 \\
\hline & 6 & +++ & 90 & - & 0 \\
\hline & 7 & +++ & 80 & - & 0 \\
\hline & 8 & +++ & 90 & - & 0 \\
\hline \multirow[t]{3}{*}{ Colon mucosa } & 1 & ++ & 50 & - & 0 \\
\hline & 2 & - & 0 & - & 0 \\
\hline & 3 & ++ & 50 & - & 0 \\
\hline \multirow[t]{3}{*}{ Colon musculature } & 1 & + & 50 & - & 0 \\
\hline & 2 & + & 50 & - & 0 \\
\hline & 3 & ++ & 40 & - & 0 \\
\hline \multirow[t]{9}{*}{ Colon carcinoma } & 1 & ++ & 90 & + & 100 \\
\hline & 2 & +++ & 90 & + & 100 \\
\hline & 3 & + & 90 & - & 0 \\
\hline & 4 & +++ & 90 & - & 0 \\
\hline & 5 & ++ & 90 & - & 0 \\
\hline & 6 & +++ & 90 & - & 0 \\
\hline & 7 & ++ & 90 & - & 0 \\
\hline & 8 & ++ & 90 & - & 0 \\
\hline & 9 & +++ & 90 & - & 0 \\
\hline \multirow[t]{4}{*}{ Endometrium } & 1 & + & 50 & - & 0 \\
\hline & 2 & + & 80 & - & 0 \\
\hline & 3 & ++ & 60 & - & 0 \\
\hline & 4 & ++ & 60 & - & 0 \\
\hline \multirow[t]{4}{*}{ Epididymis } & 1 & +++ & 80 & + & 100 \\
\hline & 2 & ++ & 50 & + & 100 \\
\hline & 3 & +++ & 90 & - & 0 \\
\hline & 4 & +++ & 90 & + & 100 \\
\hline \multirow[t]{4}{*}{ Heart } & 1 & - & 0 & - & 0 \\
\hline & 2 & - & 0 & - & 0 \\
\hline & 3 & - & 0 & - & 0 \\
\hline & 4 & - & 0 & - & 0 \\
\hline \multirow[t]{4}{*}{ Kidney } & 1 & + & 30 & - & 0 \\
\hline & 2 & ++ & 20 & - & 0 \\
\hline & 3 & + & 40 & - & 0 \\
\hline & 4 & + & 40 & + & 100 \\
\hline Lung & 1 & ++ & 50 & + & 50 \\
\hline & 2 & ++ & 59 & + & 50 \\
\hline & 3 & ++ & 50 & + & 50 \\
\hline & 4 & - & 0 & + & 50 \\
\hline Lymph node & 1 & ++ & 30 & - & 0 \\
\hline & 2 & ++ & 30 & - & \\
\hline Muscle skeletal & 1 & - & 0 & - & 0 \\
\hline & 2 & - & 0 & - & 0 \\
\hline & 3 & - & 0 & - & 0 \\
\hline & 4 & - & 0 & - & 0 \\
\hline Pancreas & 1 & + & 50 & - & 0 \\
\hline & 2 & + & 50 & - & 0 \\
\hline & 3 & + & 50 & - & 0 \\
\hline & 4 & + & 50 & - & 0 \\
\hline Parotidea & 1 & + & 50 & ++ & 100 \\
\hline & 2 & + & 50 & ++ & 100 \\
\hline Placenta & 1 & ++ & 50 & - & 0 \\
\hline & 2 & ++ & 50 & - & 0 \\
\hline & 3 & - & 0 & - & 0 \\
\hline & 4 & ++ & 50 & - & 0 \\
\hline & & & & (Con & nued) \\
\hline
\end{tabular}

Table 2. Continued

\begin{tabular}{|c|c|c|c|c|c|}
\hline \multirow[b]{2}{*}{ Tissue } & \multirow[b]{2}{*}{ Sample } & \multicolumn{2}{|c|}{ Nuclear } & \multicolumn{2}{|c|}{ Cytoplasm } \\
\hline & & Intensity & $\%$ & Intensity & $\%$ \\
\hline \multirow[t]{4}{*}{ Prostate } & 1 & + & 50 & - & 0 \\
\hline & 2 & + & 80 & - & 0 \\
\hline & 3 & ++ & 80 & - & 0 \\
\hline & 4 & - & 0 & - & 0 \\
\hline \multirow[t]{8}{*}{ Prostate carcinoma } & 1 & +++ & 100 & - & 0 \\
\hline & 2 & +++ & 100 & - & 0 \\
\hline & 3 & +++ & 100 & - & 0 \\
\hline & 4 & +++ & 100 & - & 0 \\
\hline & 5 & ++ & 100 & - & 0 \\
\hline & 6 & +++ & 90 & - & 0 \\
\hline & 7 & +++ & 100 & - & 0 \\
\hline & 8 & ++ & 90 & - & 0 \\
\hline \multirow[t]{4}{*}{ Skin } & 1 & ++ & 50 & - & 0 \\
\hline & 2 & ++ & 50 & - & 0 \\
\hline & 3 & ++ & 50 & - & 0 \\
\hline & 4 & ++ & 50 & - & 0 \\
\hline \multirow[t]{4}{*}{ Spleen } & 1 & ++ & 30 & - & 0 \\
\hline & 2 & + & 30 & - & 0 \\
\hline & 3 & - & 0 & - & 0 \\
\hline & 4 & + & 20 & - & 0 \\
\hline \multirow[t]{4}{*}{ Stomach } & 1 & ++ & 30 & + & 100 \\
\hline & 2 & - & 0 & - & 0 \\
\hline & 3 & ++ & 50 & + & 100 \\
\hline & 4 & - & 0 & - & 0 \\
\hline \multirow[t]{4}{*}{ Thymus } & 1 & + & 50 & - & 0 \\
\hline & 2 & ++ & 20 & - & 0 \\
\hline & 3 & + & 30 & - & 0 \\
\hline & 4 & + & 20 & - & 0 \\
\hline \multirow[t]{2}{*}{ Thyroidea } & 1 & + & 20 & - & 0 \\
\hline & 2 & + & 20 & - & 0 \\
\hline \multirow[t]{3}{*}{ Tonsil } & 1 & ++ & 50 & - & 0 \\
\hline & 2 & ++ & 50 & - & 0 \\
\hline & 3 & ++ & 50 & - & 0 \\
\hline
\end{tabular}

Table 3. RBM3 expression in a variety of CNS tumours and normal brain tissues

\begin{tabular}{|c|c|c|c|c|c|c|}
\hline \multirow[b]{2}{*}{ Tumor entity } & \multirow{2}{*}{$\begin{array}{l}\text { No. of } \\
\text { cases }\end{array}$} & \multirow{2}{*}{$\begin{array}{l}\text { Percent of } \\
\text { positive } \\
\text { nuclei } \\
\text { (SD) }\end{array}$} & \multicolumn{4}{|c|}{$\begin{array}{c}\text { Staining intensity, no. of cases } \\
\text { (percent) }\end{array}$} \\
\hline & & & +++ & ++ & + & 0 \\
\hline $\begin{array}{r}\text { Glioblastoma } \\
\text { multiforme }\end{array}$ & 37 & $71( \pm 23)$ & 23 & 11 & $3(8)$ & $0(0)$ \\
\hline Astrocytoma & 48 & 8) & $12(25)$ & $18(37)$ & $11(23)$ & $7(15)$ \\
\hline Oligodendroglioma & 22 & 8 & $9(41)$ & $8(36)$ & $3(14)$ & $2(9)$ \\
\hline Meningioma & 48 & 36( & $1(2)$ & $15(31)$ & $26(54)$ & $6(13)$ \\
\hline Neurofibr & 34 & ) & $28(82)$ & $5(15)$ & $1(3)$ & $0(0)$ \\
\hline Schwannon & 46 & $49(=$ & $23(50)$ & $18(39)$ & $5(11)$ & $0(0)$ \\
\hline Normal brain & 10 & $5( \pm 5)$ & $0(0)$ & $0(0)$ & $5(50)$ & $5(50)$ \\
\hline
\end{tabular}

tion rate (Fig. 1A). This effect was observed irrespective of whether HEK293 cells were seeded in complete medium (containing 10\% FCS) or in starving medium (containing $0.1 \%$ FCS) (Fig. 1A). However, prolonged incubation of HEK293 cells in fresh medium resulted in a decreased cell proliferation rate secondary to contact inhibition and prolonged incubation of HEK293 cells in starving medium stopped proliferation secondary to cell quiescence. Both conditions were paralleled by marked RBM3 mRNA and protein down-regulation (Fig. 1A). HEK293 cells showed no signs of caspase- 3 activation up to $5 \mathrm{~d}$ of contact inhibition or starva- 

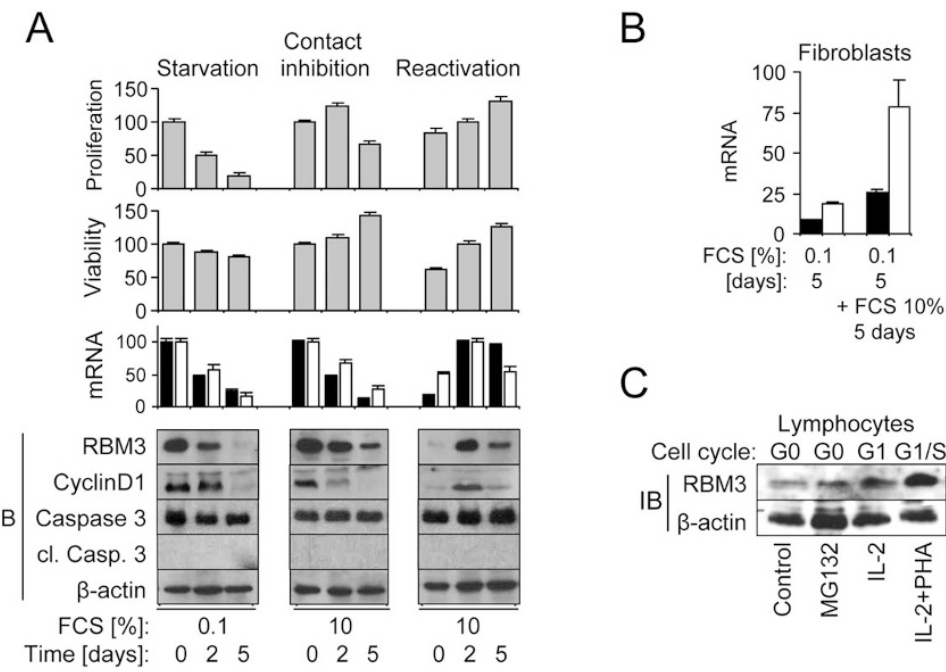

Figure 1. RBM3 expression correlates with cell proliferation. (A) Starvation: HEK293 cells were grown $2 \mathrm{~d}$ in complete medium (10\% FCS), washed twice with PBS, and a fresh starving medium $(0.1 \%$ FCS $)$ was added (day $0=100 \%)$. Contact inhibition: HEK293 cells were synchronized for $2 \mathrm{~d}$ in starving medium and then fresh complete medium was added (day $0=100 \%$ ). Reactivation: HEK293 cells were starved for 5 d, seeded in new plates (day 0 ), and grown in complete medium as indicated (day $3=100 \%$ ). Viability (MTS assay), proliferation rates (BrdU assay), and mRNA expression (real-time RT-PCR) of RBM3 $\square$ and CyclinD1 $\square$ were assessed as indicated. Columns represent mean values plotted from triplicate determinations with denoting standard deviations. Immunoblotting (IB) was performed for the indicated antigens, Caspase-3 (Cleaved Caspase-3). (B) mRNA of RBM3 $\square$ and CyclinD1 $\square$ was quantified either in quiecent fibroblasts (MRC-5 cells), which were grown for $5 \mathrm{~d}$ in starving medium (first couple of columns) or in proliferating fibroblasts grown in complete medium (second couple). (C) After incubation of peripheral lymphocytes with the proteasome inhibitor MG132 (10 $\mu \mathrm{M}), \mathrm{IL}-2$ (100,000 U/mL), or both IL-2 and PHA $(0.01 \mathrm{mg} / \mathrm{mL})$, cell cycle was assessed, and RBM3 and $\beta$-actin protein were detected by IB.



Figure 2. Immunohistochemistry for RBM3. Brown stain demonstrates the location of the RBM3 protein in normal colon mucosa $(A)$ and colon adenocarcinoma $(B)$ as well as normal prostate $(C)$ and prostate adenocarcinoma $(D)$. Magnification $\times 630$. Scale bar, $100 \mu \mathrm{m}$.

tion, and there was no impaired proliferation capacity when cells were split and seeded in fresh complete medium after $5 \mathrm{~d}$ (reactivation, Fig. 1A). Similar results were found when fibroblasts were used (Fig. 1B).

To further investigate the observed direct relationship between proliferation and RBM3 expression, we collected peripheral circulating human lymphocytes displaying the G0/G1 flow cytometry pattern of resting cells. Although addition of IL-2 or of the inhibitor of proteasomal degradation, MG132, did not lead to a re-entry into cell cycle and had no effect on RBM3 expression, the addition of PHA greatly forced re-entry of lymphocytes into cell cycle and was associated with strong RBM3 up-regulation (Fig. 1C).

RBM3 staining is strong in malignant and nonmalignant proliferating cells. To investigate whether RBM3 expression is related to cell proliferation in human tissue samples, we investigated RBM3 expression in a multitissue array including normal tissues and tumor tissues of multiple organs (Table 2). Tumor cells, irrespective of their origin, displayed in general strong RBM3 staining. Nuclear RBM3 staining exceeded cytoplasmatic staining in all normal and tumor samples except in parotid gland. No RBM3 expression was found in heart and skeletal muscle tissues. In general, normal tissues compared with malignant tissues expressed RBM3 at a much lower level of staining intensity. Moreover, the percentage of cells stained positive for RBM3 was much lower in normal, compared with malignant tissue. However, nonmalignant proliferating cells, such as intestinal epithelial cells, showed also high RBM3 expression (Fig. 2). The only exception of normal tissue staining with general strong intensity in a high percentage of cell nuclei was epididymis (Table 2). In CNS tumors, nuclear staining intensity was lower and present in a smaller percentage of cells in slowly growing tumors, especially in meningioma and oligodendrogliomas, as opposed to a high percentage of tumor cell nuclei with strong expression in most samples of glioblastoma multiforme (Table 3). Cytoplasm was almost uniformly negative.

RBM3 rescues cells from cell death caused by serum starvation. As serum-starved cells decrease proliferation and RBM3 expression in parallel, we asked how artificial overexpression of RBM3 might affect cell survival under various cell culture conditions. We stably over-expressed RBM3 in HEK293 cells, with a similar partitioning of RBM3 between 
A

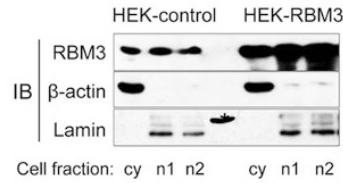

C

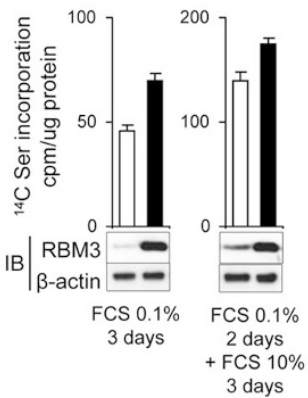

$\mathrm{B}$



Figure 3. Over-expression of RBM3 protects HEK 293 cells from loss of viability caused by serum starvation. (A) HEK293 cells with the empty pCEP4 vector (HEK-control) and RBM3 over-expressing HEK293 cells (HEK-RBM3) were starved as described in Figure 1, starving medium was changed after 2 and 4 d as indicated. By use of the MTS assay and appropriate cell dilution series, cell numbers of proliferating cells were calculated per mL. Columns, mean of quadruplicates; bars, \pm SD mRNA expression of CyclinD1 was determined by real-time RT-PCR, bars denote the mean \pm SD. Immunoblotting (IB) was performed for the indicated antigens. $(B)$ Translation rate was measured by ${ }^{14} \mathrm{C}$ serine incorporation relative to total protein concentration in various HEK-RBM3 $\square$ and HEK-control cells $\square$. Columns, mean of triplicates; bars, \pm SD. IB was performed for the indicated antigens. (C) Nuclear extracts of HEK-control and HEK-RBM3 cells were prepared with stringent (n1) and very stringent conditions (n2). IB was performed for the indicated antigens, and $\beta$-actin served as marker for the cytoplasmatic (cy) and lamin for the nuclear cell fraction. The $100 \mathrm{kD}$ reference band is marked by an asterisk. All data shown are representative of at least three independent experiments.
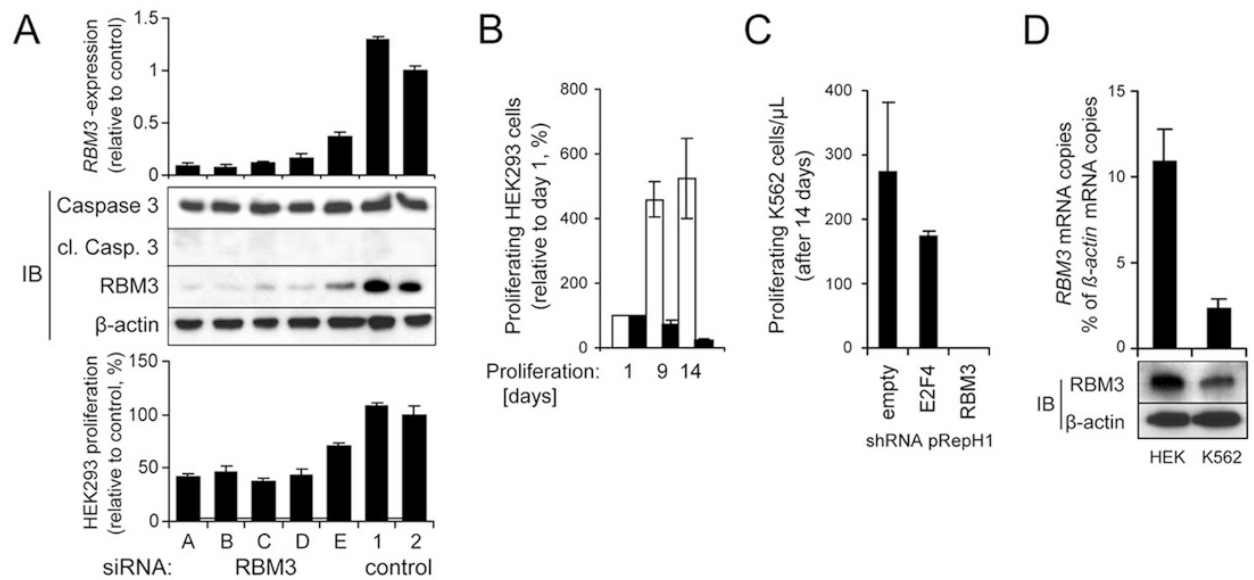

Figure 4. Down-regulation of RBM3 inhibits cell proliferation and finally leads to cell death without activation of caspase-2. (A) HEK293 cells were treated with various RBM3 specific siRNAs (RBM3 A, B, C, D, and E). RBM3 mRNA expression is given relative to control siRNA-2 as quantitated by real-time RT-PCR $24 \mathrm{~h}$ after transfection. Immunoblotting (IB) for the indicated antigens was done $72 \mathrm{~h}$ after transfection, cleaved caspase-3 (cleaved Caspase-3). HEK293 proliferation rate was measured $96 \mathrm{~h}$ after transfection by MTS tetrazolium assay. ( $B$ and $C$ ) Proliferation rate as measured by MTS assay decreases strongly in HEK293 ( $B$ ) and K562 cells $(C)$ constitutively expressing RBM3 specific shRNAs ( $B \boldsymbol{G}$ and $C$ as indicated) in contrast to controls ( $B$, cells constitutively expressing control E2F4-shRNA $\square$ and $C$ as indicated). (D) RBM3 mRNA copies relative to copies of $\beta$-actin copies and protein expression as indicated in wild-type proliferating HEK293 and K562 cells, IB. All data shown are representative of at least three independent experiments and columns, mean of triplicates, bars, \pm SD.

nucleus and cytoplasm as in wild-type cells (Fig. 3A). HEK293 cells with stable over-expression of RBM3 survived severe serum starvation much better than HEK293 cells expressing the empty vector (Fig. $3 B$ ).

RBM3 increases protein synthesis under starving conditions. Up-regulation of RBM3 was recently found to enhance global translation (6). Therefore, we investigated the uptake of radioactively labeled serine and phenylalanine in RBM3overexpressing HEK293 cells. Under optimal culture conditions (10\% FCS), there was no increase of amino acid uptake associated with artificial RBM3 over-expression. However, RBM3-overexpressing HEK293 cells had significantly higher amino acid incorporation when these cells were subjected to serum starvation, compared with starved controls (Fig. 3C).

RBM3 is indispensible for cell proliferation. We designed a variety of siRNAs targeting multiple sequence areas of RBM3 mRNA that transiently down-regulate endogenous RBM3 mRNA and protein (Fig. 4A). siRNAs that transiently knocked down RBM3 also reduced cell proliferation and viability within $72 \mathrm{~h}$, compared with control siRNAs (Fig. 

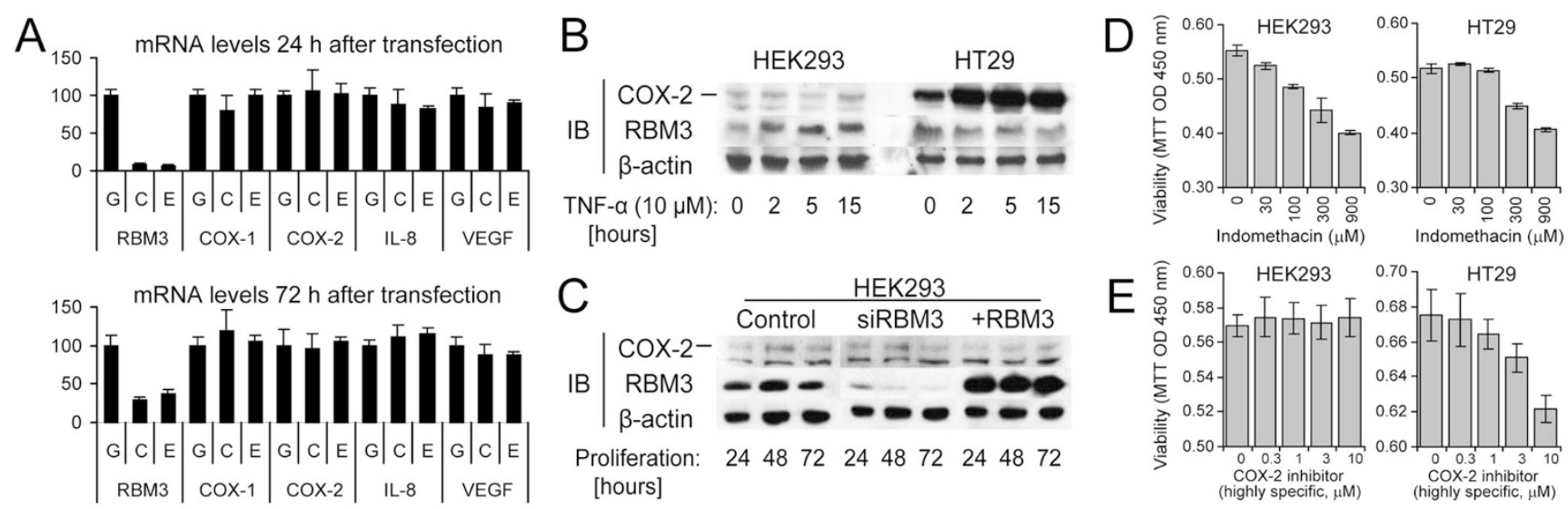

Figure 5. In HEK293 cells neither RBM3 down-regulation nor RBM3 over-expression affects the expression levels of COX-1, COX-2, IL-8, and VEGF. (A) $24 \mathrm{~h}$ and $72 \mathrm{~h}$ after transfection of HEK293 cells with control siRNA (G, targeting GFP) or two different siRNAs targeting RBM3 (C and E) mRNA expression of $C O X-1, C O X-2, I L-8$, and $V E G F$ was determined by real-time RT-PCR. Columns, mean values plotted from triplicate determinations, and values are given relative to the expression level of $\mathrm{G}(\mathrm{G}=100 \%)$; bars, \pm SD $(B)$ HEK293 and HT29 cells were treated as indicated with TNF- $\alpha$, and immunoblotting (IB) was performed for the indicated antigens. (C) HEK293 cells were transfected with control siRNA targeting GFP (control), with siRNA targeting RBM3 (siRBM3), or with the RBM3 expression vector RBM3-pCEP4 (+RBM3). At various time points after transfection, IB was performed for the indicated antigens. $(D)$ Both cell lines, HEK293 and HT29, were incubated with indomethacin, or the COX-2 specific inhibitor, indomethacin heptyl ester as indicated. After 96 h, cell viability was measured by the use of the MTS tetrazolium assay. Columns, mean of quadruplicates; bars, \pm SD. All data shown are representative of at least three independent experiments.

4A). Cell cycle analysis failed to reveal apoptotic cells (sub-G1 fraction) at various time points after siRNA transfection, even when cells were transfected repeatedly (data not shown). Loss of viability was not associated with caspase-3 activation (Fig. 4A). We then cloned these siRNA sequences into shRNA expression vectors and transfected them into HEK293 or K562 cells. shRNA-mediated permanent RBM3 knockdown resulted in profound loss of cell viability in both cell types (HEK293 and K562 in Fig. 4B and C, respectively), albeit RBM3 mRNA and protein expression was approximately three times lower in K562, compared with HEK293 cells (Fig. 4D).

RBM3 action in HEK293 cells does not involve COX-2, $\boldsymbol{V E G F}$, or $\boldsymbol{I L - 8}$. These cytokines VEGF and IL-8 as well as COX-2 have been implicated to act downstream of RBM3 in regulating cell survival in colon carcinoma cells (9). This prompted us to investigate the interplay of RBM3 and COX-2, VEGF, and IL-8 in HEK293 cells. Artificial manipulation of RBM3 expression, i.e., over-expression or silencing, did not change the mRNA and protein expression pattern of COX-2, VEGF, IL-8, or COX-1 in HEK293 cells (Fig. 5A and $C$ ). HEK293 did not show COX-2 induction upon incubation with $\mathrm{TNF} \alpha$ while human colon carcinoma HT29 cells strongly up-regulated COX-2 protein (Fig. 4B). In contrast, RBM3 protein expression slightly increased in HEK293 but decreased in HT29 cells upon $\mathrm{TNF} \alpha$ treatment. Incremental increases of the nonspecific cyclooxygenase inhibitor, indomethacin, reduced cell viability in both HEK293 and HT29 cells (Fig. $5 D$ ), whereas the COX-2 specific inhibitor, indomethacin heptyl ester, dose-dependently reduced cell viability in HT29 but not in HEK293 cells (Fig. 5E).

\section{DISCUSSION}

Control of cellular maintenance requires orchestration of multiple molecular players on all levels of gene expression.
RNA-binding proteins influence the structure and interactions of RNAs and play critical roles in their biogenesis, stability, function, transport, and cellular localization by a variety of mechanisms (17). So far, two different mechanisms have been ascribed to RBM3 to exert its action. First, over-expression of RBM3 in mouse neuroblastoma N2a cells has been shown to induce global translation presumably by decreasing microRNA levels (6). Second, down-regulation of RBM3 in human HCT116 colon cancer cells leading to mitotic catastrophe was associated with a loss in the translation of the mRNAs for COX-2, VEGF, and IL-8 (9).

In the experiments described here, we demonstrate that 1) RBM3 is over-expressed in different tumors compared with normal tissues, 2) RBM3 expression parallels cell proliferation in various cell types in vitro and in vivo, and (3) RBM3 is pivotal for cell survival at least under adverse growth conditions.

Among the so far described mechanisms attributed to RBM3 action, we found evidence for RBM3 involvement in global translation within our cell system. However, the survival-promoting activity of RBM3 does not require COX-2, at least not in HEK293 cells, as suggested from the following three observations: First, neither RBM3 over-expression nor RBM3 down-regulation affects COX-2 mRNA and protein levels in HEK293 cells. Second, proliferation of HEK293 cells is strongly perturbed when RBM3 expression is manipulated but there is no effect when a specific COX-2 inhibitor is used (in contrast to human colon cancer HT29 cells). Third, although artificial down-regulation of RBM3 did not affect the classical apoptosis pathway in HEK293 cells, there was a significant change in translation rate indicating that RBM3 loss may lead to a lack of important proteins required for cell growth.

RBM3 was first described as a cold shock protein upregulated in response to decreased ambient temperature (3). 
We later showed that moderate hypoxia was sufficient to induce RBM3 (2). It may be speculated that RBM3 is required for maintenance of cellular integrity launching a cellular backup mechanism. Hypothermia as well as hypoxia lead to a general inhibition of protein synthesis $(18,19)$, and RBM3 seems to be required to preserve ribosomal mRNA loading and hence translation from a subpopulation of mRNAs (6).

In addition to hypothermia and hypoxia, data from two independent genome-wide gene expression analyses aimed to profile the transcriptional program of human fibroblasts in the response to serum indicate a much broader role for RBM3 $(20,21) . R B M 3$ transcripts were found to be low in quiescent cells and significantly increased after induction of cell proliferation by serum stimulation. This effect was found to be not cell cycle associated (21). In addition, RBM3 transcripts were reportedly down-regulated in growth-arrested T98G cancer cells caused by either serum deprivation or contact inhibition (22). In all of our experiments with proliferating or starving HEK293 and K562 cells, fibroblasts, and primary lymphocytes, RBM3 mRNA and protein levels paralleled markers of proliferation. Moreover, we found stronger staining of RBM3 in cells of normal tissues with high proliferation rate, e.g., intestinal epithelial cells, compared with resting cells, e.g., intestinal stromal cells and skeletal and heart muscle cells. Strong staining was seen in all cancer samples. This puts forward the model that RBM3 acts as an on-off or off-on switch in resource-poor conditions such as hypothermia or hypoxia and serum starvation. A first approach toward clinical implementation may be the recent findings of Zeng et al. (23), suggesting that down-regulating of cold inducible proteins including RBM3 in prostate cancer cells by exposure to therapeutical hyperthermia may render cancer cells more susceptible to therapy. However, additional work is required to establish RBM3 as a new target in cancer diagnosis and therapy especially by investigating the molecular mechanisms that promote RBM3 expression under adverse conditions and in cancer cells.

Acknowledgments. We are grateful to Barbara Stalder, Molecular Pathology Section, Institute of Pathology, Basel University Hospital, for production of the tissue array and excellent performance of immunohistochemical procedures. We thank Dr. Terttu Suormala for expert technical assistance in determination of protein synthesis, Metabolic Unit, University Children's Hospital, Basel.

\section{REFERENCES}

1. Derry JM, Kerns JA, Francke U 1995 RBM3, a novel human gene in Xp11.23 with a putative RNA-binding domain. Hum Mol Genet 4:2307-2311
2. Wellmann S, Buhrer C, Moderegger E, Zelmer A, Kirschner R, Koehne P, Fujita J, Seeger K 2004 Oxygen-regulated expression of the RNA-binding proteins RBM3 and CIRP by a HIF-1-independent mechanism. J Cell Sci 117:1785-1794

3. Danno S, Nishiyama H, Higashitsuji H, Yokoi H, Xue JH, Itoh K, Matsuda T, Fujita J 1997 Increased transcript level of RBM3, a member of the glycine-rich RNAbinding protein family, in human cells in response to cold stress. Biochem Biophys Res Commun 236:804-807

4. Danno S, Itoh K, Matsuda T, Fujita J 2000 Decreased expression of mouse Rbm3, a cold-shock protein, in Sertoli cells of cryptorchid testis. Am J Pathol 156:16851692

5. Williams DR, Epperson LE, Li W, Hughes MA, Taylor R, Rogers J, Martin SL, Cossins AR, Gracey AY 2005 Seasonally hibernating phenotype assessed through transcript screening. Physiol Genomics 24:13-22

6. Dresios J, Aschrafi A, Owens GC, Vanderklish PW, Edelman GM, Mauro VP 2005 Cold stress-induced protein Rbm3 binds 60S ribosomal subunits, alters microRNA levels, and enhances global protein synthesis. Proc Natl Acad Sci USA 102:18651870

7. Kita H, Carmichael J, Swartz J, Muro S, Wyttenbach A, Matsubara K, Rubinsztein DC, Kato K 2002 Modulation of polyglutamine-induced cell death by genes identified by expression profiling. Hum Mol Genet 11:2279-2287

8. Cok SJ, Acton SJ, Sexton AE, Morrison AR 2004 Identification of RNA-binding proteins in RAW 264.7 cells that recognize a lipopolysaccharide-responsive element in the 3-untranslated region of the murine cyclooxygenase-2 mRNA. J Biol Chem 279:8196-8205

9. Sureban SM, Ramalingam S, Natarajan G, May R, Subramaniam D, Bishnupuri KS, Morrison AR, Dieckgraefe BK, Brackett DJ, Postier RG, Houchen CW, Anant S 2008 Translation regulatory factor RBM3 is a proto-oncogene that prevents mitotic catastrophe. Oncogene 27:4544-4556

10. Buhrer C, Berlin C, Thiele HG, Hamann A 1990 Lymphocyte activation and expression of the human leucocyte-endothelial cell adhesion molecule 1 (Leu-8/TQ1 antigen). Immunology 71:442-448

11. Wellmann S, Bettkober M, Zelmer A, Seeger K, Faigle M, Eltzschig HK, Buhrer C 2008 Hypoxia upregulates the histone demethylase JMJD1A via HIF-1. Biochem Biophys Res Commun 372:892-897

12. Shalapour S, Zelmer A, Pfau M, Moderegger E, Costa-Blechschmidt C, van Landeghem FK, Taube T, Fichtner I, Buhrer C, Henze G, Seeger K, Wellmann S 2006 The thalidomide analogue, CC-4047, induces apoptosis signaling and growth arrest in childhood acute lymphoblastic leukemia cells in vitro and in vivo. Clin Cancer Res 12:5526-5532

13. Janz M, Hummel M, Truss M, Wollert-Wulf B, Mathas S, Johrens K, Hagemeier C, Bommert K, Stein H, Dorken B, Bargou RC 2006 Classical Hodgkin lymphoma is characterized by high constitutive expression of activating transcription factor 3 (ATF3), which promotes viability of Hodgkin/Reed-Sternberg cells. Blood 107:2536-2539

14. Willard HF, Ambani LM, Hart AC, Mahoney MJ, Rosenberg LE 1976 Rapid prenatal and postnatal detection of inborn errors of propionate, methylmalonate, and cobalamin metabolism: a sensitive assay using cultured cells. Hum Genet 34:277283

15. Gerstner B, Buhrer C, Rheinlander C, Polley O, Schuller A, Berns M, Obladen M, Felderhoff-Mueser U 2006 Maturation-dependent oligodendrocyte apoptosis caused by hyperoxia. J Neurosci Res 84:306-315

16. Kononen J, Bubendorf L, Kallioniemi A, Barlund M, Schraml P, Leighton S, Torhorst J, Mihatsch MJ, Sauter G, Kallioniemi OP 1998 Tissue microarrays for high-throughput molecular profiling of tumor specimens. Nat Med 4:844-847

17. Glisovic T, Bachorik JL, Yong J, Dreyfuss G 2008 RNA-binding proteins and post-transcriptional gene regulation. FEBS Lett 582:1977-1986

18. Burdon RH 1987 Temperature and animal cell protein synthesis. Symp Soc Exp Biol 41:113-133

19. Hochachka PW, Buck LT, Doll CJ, Land SC 1996 Unifying theory of hypoxia tolerance: molecular/metabolic defense and rescue mechanisms for surviving oxygen lack. Proc Natl Acad Sci USA 93:9493-9498

20. Iyer VR, Eisen MB, Ross DT, Schuler G, Moore T, Lee JC, Trent JM, Staudt LM, Hudson J Jr, Boguski MS, Lashkari D, Shalon D, Botstein D, Brown PO 1999 The transcriptional program in the response of human fibroblasts to serum. Science 283:83-87

21. Chang HY, Sneddon JB, Alizadeh AA, Sood R, West RB, Montgomery K, Chi JT, van de Rijn M, Botstein D, Brown PO 2004 Gene expression signature of fibroblast serum response predicts human cancer progression: similarities between tumors and wounds. PLoS Biol 2:E7

22. Cam H, Balciunaite E, Blais A, Spektor A, Scarpulla RC, Young R, Kluger Y, Dynlacht BD 2004 A common set of gene regulatory networks links metabolism and growth inhibition. Mol Cell 16:399-411

23. Zeng Y, Kulkarni P, Inoue T, Getzenberg RH 2009 Down-regulating cold shock protein genes impairs cancer cell survival and enhances chemosensitivity. J Cell Biochem 107:179-188 